\title{
Pillars and pathways: foundations of recovery in Irish forensic mental health care
}

\begin{abstract}
The Central Mental Hospital in Ireland is one of the oldest forensic mental health units in Europe. The hospital is currently in the process of transforming from a single inpatient site to a modern national forensic mental health service. Central to this transformation is the need to move from the traditional security-focused model of care to a model of recovery. The challenge incumbent within this transformation is to incorporate a sophisticated amalgamation of the patients' needs while recognising the broad range of security requirements in a forensic setting. This paper considered that adopting an integrated care pathway (ICP) approach would provide the service with a vehicle to re-engineer our principles and systems of care. Likewise we hypothesised that the ICP would enable us to consolidate best practices such as multidisciplinary working, structured professional judgement and the involvement of the patient and their carers. Thus far it has afforded us the opportunity to examine many aspects of the care delivered within the service. It has provided a shared understanding of key standards among clinicians, service users and carers that are necessary to implement a quality care pathway. It has certainly not been a stagnant process, and the initial work often bears no resemblance to the current process. In turn, we expect that it will continue to change as the path travelled is as important as the outcome and the ICP becomes a dynamic part of the organisation.
\end{abstract}

\section{KEY WORDS}

forensic mental health; integrated care pathways; pillars of care

\section{Introduction}

The concept of integrated clinical pathways has been employed within health care since the 1980s
(Zander, 2002). Their use has been considered to identify cost effectiveness (Riley, 1998), length of stay and quality outcomes (Kent \& Chalmers, 
2006). Research in this area has, however, been focused primarily on general health care. Their use in mental health and forensic mental health has been less widespread (Green, 2008). To introduce such pathways successfully, a number of key foundations are identified in the literature as necessary to develop a quality care pathway. They include identification of local champions and strong leadership, the support of senior management, service user involvement and active multidisciplinary participation (De Bleser et al, 2006; Green, 2008).

Likewise, the notion of introducing any model of care in a secure environment is considered fraught with challenges (Gralton et al, 2006) due to the complex nature of the needs and risks posed by this group of individuals, and the comparatively long periods of time spent as an inpatient (Badger et al, 1999).

This project began in late 2007, following on from a comprehensive needs analysis of the characteristics of the target patient group (O'Neill et al, 2003), and a restructuring of both the clinical team and the service into designated clusters of care; see Table 1, below.

The development of care clusters enabled the service to identify the level of recovery and rehabilitation capacity for each patient within the service and triggered the process to develop a patient-focused care pathway. At the same time, Irish mental health services have been inundated with a variety of legislative and policy changes that have required us to update our clinical practice to contemporary standards. In 2006, the Department of Health introduced its strategy for mental health services in its Vision for Change document (DoHC, 2006). The central tenet of this new model for care was to be a comprehensive, multi-disciplinary team approach, providing continuity of care, more effective co-ordination and greater emphasis on service user involvement, advocacy and personal responsibility for recovery. Lamont et al (2009) commented on this need to engage all stakeholders in the process of care, adding that anyone who is affected by the collective decisions of others should be recognised as a key stakeholder. This theory regarding all stakeholders being involved in the patients care becomes central to the notion of integrated care pathways (Hayward-Rowse $\&$ Whittle, 2006).

The two relevant legislative frameworks under which the service operates, the Mental Health Act (2001) and the Criminal Law Insanity Act (2006), also influenced the formation of this pathway. The service still operated single-discipline documentation which reflected single-disciplinary practice. Both the Mental Health Commission and the service recognised that the use of a single composite set of documentation would better reflect and induce patient-centred care where the notes belong to the patient (Mental Health Act, 2006).

Indeed, it has been considered that using integrated documentation can have the additional benefits of enhancing inter-professional collaboration and reducing professional isolation (Atwall \& Caldwell, 2002). It is recognised that modern health care has become more complex (Green, 2008) and that is nowhere more evident

Table 1: Initial clustering of services

\begin{tabular}{lll}
\hline Cluster & Clinical function & Security function \\
\hline Pre-admission & $\begin{array}{l}\text { Court diversion } \\
\text { Prison in-reach } \\
\text { Mainstream mental health }\end{array}$ & High \\
& $\begin{array}{l}\text { Admission and assessment } \\
\text { Acute }\end{array}$ & \\
Medium & $\begin{array}{l}\text { Challenging behaviour } \\
\text { Continuing care }\end{array}$ & Medium \\
& $\begin{array}{l}\text { Long stay } \\
\text { Rehabilitation }\end{array}$ & \\
Recovery and rehabilitation & $\begin{array}{l}\text { Hostel } \\
\text { Day centre } \\
\text { Community outreach }\end{array}$ & \\
& &
\end{tabular}




\section{Pillars and pathways: foundations of recovery in Irish forensic mental health care}

than in forensic mental health care, where professionals and patients navigate through the two systems of health and justice (Adshead \& Sarkar, 2005). The service had already spent a considerable degree of time and expense on assimilating a range of professionals and protocols to address clinical need, broader health care needs (Camberwell Assessment of Need Forensic (CANFOR); Thomas et al, 2003) and structured risk assessment and management strategies. These included the Threshold Assessment Grid, Dynamic Assessment of Situational Aggression, Historical Clinical Risk20 and Suicide Risk Assessment Management Manual (Webster et al, 1997; Slade et al, 2000; Bouch \& Marshall, 2003; Ogloff \& Daffern, 2006; Thomas et al, 2003). Concurrently these professional approaches had been complemented by the establishment of an active carers group, patients' forum and advocacy service.

Thus many of the foundations identified in the literature review as necessary for the development of a quality care pathway (ICP) were in place. The challenge was then how to bring all these different stakeholders, practices and expectations into a co-ordinated and integrated journey of care that met best practice and the needs of the patient and society.

\section{The project}

This project was executed over five cycles commencing in late 2007. An initial steering group was set up to conduct a literature review to establish current best practice in relation to documentation and effective introduction of a pathway. An action research approach was adopted that acknowledged that the successful implementation of the ICP would require the involvement and ownership of all stakeholders.

Participatory action research is considered to be contingent on authentic participation which involves a continuing spiral of planning, acting (implementing plans), observing (and then re-planning and so around the spiral again) (Kalliola, 2009). The action research process usually involves working with groups collecting initial data in an area of interest, then reflecting on the information and planning changes. One of the central tenets of this type of research is the benefit of starting small and developing a small cycle of planning, acting, observing and reflecting. This can help to define issues, ideas and assumptions more clearly, so that those involved can define more powerful questions for themselves as their work progresses (Palmer, 2009).
Following an extensive literature review and consultation with key stakeholders, there was agreement that the design of an ICP should help move clients through the treatment system and act as a guide to individual care planning. The care pathway should form all or part of the clinical record, and document clearly the care given. It was also acknowledged that variations from best practice and gaps in the care or unmet need would be identified and recorded in order to develop a culture of continuous quality improvement. More important, the care pathway should be recoveryfocused for the individual service user, based on their assessed need and risk, and include minimum standards that can be audited. A decision was made not to progress down pathways for syndromes at this particular juncture.

\section{Ethical approval}

Permission was provided by the senior management team. All involvement of patients in focus groups was conducted with due consideration of their mental capacity and ability to consent. All were at an appropriate stage of their treatment. All participants were given the opportunity to engage in or disengage from the process. Time was further allowed to discuss this before the focus groups, and in order to promote informed and uncoerced consent.

\section{Cycle I - steering group}

A multi-disciplinary steering group was established, which formed the consistent core of this project. The success of the project was, however, heavily dependent on the skills and involvement of a range of different staff who contributed extensively to this process.

The steering group initially looked at the pathway for patients accessing this service through the pre-admission and admission stages, and into the acute cluster stages. This entailed examination of the patients' journey in minute detail through each part of these stages. Following a number of consultation and educational workshops, a draft pre-admission protocol and admission document was developed and piloted on the male admission unit.

During the review of these early stages the following key areas were prioritised, and that needed further discussion and clarification. They included:

- developing and agreeing a shared understanding of standards of care that was holistic and supported recovery 


\section{Pillars and pathways: foundations of recovery in Irish forensic mental health care}

- how to incorporate risk assessment and risk management into the ICP

- meaningful user involvement

- the role of each discipline and use of evidencebased tools.

This early draft of the ICP incorporated development of three books, which would make up the beginning of the patient's pathway and set of integrated notes. Book 1 provided a framework for the pre-admission assessment for all service users before their admission, and included key clinical standards. Book 2 included the initial reception, medical and nursing needs, and risk assessment. Book 3 introduced a single document for all disciplines to record their ongoing contact with the service users.

Clinical assessment tools that had good reliability and validity were introduced by each profession. Protocols for assessment of risk of violence on admission and to assess the security level for each patient were also identified by the staff and in the literature search. They were subsequently built into this documentation.

\section{Cycle 2 - pilot project}

A pilot project was commenced to evaluate this initial work. The documents were widely circulated and a number of consultation workshops were conducted to assess face content validity. The documents were also assessed by the clinical director and director of nursing as an expert panel that was at least one step removed from the process. The steering group was also grateful that we had easy access to work carried out in this area by our international colleagues, particularly in Wales, Canada, England, Australia and Scotland. Concurrently the service had also joined the Royal College of Psychiatrist's Quality Network for Forensic Mental Health Services, which allowed us to benchmark our quality standards by international comparisons.

Nursing staff on the units were asked to keep a diary of issues or difficulties that they identified with the new documentation, and members of the steering group attended weekly ward rounds for discussion and feedback. Feedback in relation to the MDT singular documentation was very positive from all disciplines. Atwall and Caldwell (2002) contend that integrated documentation is a necessity for collaboration and ensures that care is co-ordinated. Feedback from ICP workshops in the service supported this argument, and professionals from across the disciplines noted a reduction in duplication of notes since the introduction of ICP.
The documents were then piloted over a 12 -month period by our colleagues working in the pre-admission and acute clusters, and we are indebted to their active involvement and feedback in this process.

The pre-admission assessment documents were agreed with minor changes. A report format was agreed using the Mental State Examination, the Threshold Assessment Grid (TAG), a triage instrument and a security rating grid. Standards were set by the team that ensured this information was kept up to date and provided to the admitting team before admission. Several issues were identified with the reception and admission document, including the demographic details. One of the main issues highlighted the difficulties of having the nursing and medical assessment within one book, thus inhibiting recording of joint assessments. This subjected the service user to repeating the same or similar information. Feedback from the service user focus group identified that their priority on admission was to feel safe and become orientated to their new surroundings. The process of independent admission practices by the medical and nursing team increased their stress levels, and indeed may well be considered as contradicting the current literature on risk management (Madden, 2007).

The pilot project also highlighted some areas of unnecessary duplication of information, some areas of unmet needs and previously set standards that could not be achieved within existing resources. This entailed further discussion with heads of each discipline, and the development of the pathway by writing a time-task matrix for each milestone. This matrix has been espoused in the literature as being imperative to decide (Green, 2008):

- what needs doing

- why it needs doing

- how will it be done

- by whom.

Key clinical standards were then identified for each of the following milestones:

- pre-admission reports

- check of legal orders

- nursing assessment within two hours

- medical assessment within four hours

- seen by consultant within 24 hours

- allocation of consultant, primary nurse and key worker

- information folder to patient within 72 hours by social worker

- seen by other disciplines within one week 


\section{Pillars and pathways: foundations of recovery in Irish forensic mental health care}

- ward round within seven days and weekly thereafter

- structured risk assessment by first case conference

- case conference within one month, or 14 days if fitness to plead timescale

- discharge or transfer to medium cluster.

These standards were subsequently structured into the following patient's clinical file:

- pre-admission report

- Book 1 - Nursing Admission

- Book 2 - Medical Admission

- Book 3 - Progress Notes Book

- Book 4 - Individual Care Plan

- Book 5 - DASA

- reports and investigations.

Allocation of a named nurse and consultant are evidentially essential elements in the co-ordination of anyone's health care and a standard being set by the Health Service Executive and Mental Health Commission (DoHC, 2006; MHC, 2009). Likewise the need for the patient to be seen by a consultant within 24 hours has been mandated by our mental health legislation, as is the need for the patient to have a copy of their legal rights and information on their care (MHC, 2009). One of the additional benefits of the ICP process is the ability to configure such standards into our clinical practice. This is then reflected in our documentation, which in turn leads the clinician through each standard in a structured manner.

At the same time the clinician's professional judgement remains the central tenet for delivery of effective and high-quality health care. The pathway then acts as a template of care that is expected for patients within the service. Each clinician should evaluate and determine whether the defined intervention(s) is appropriate for the patient's individual needs. Such determinations should be made using validated, empirically grounded assessment tools or guidelines, and in conjunction with the patient. This process should be done systematically in order to allow for assessment, formulation and management of care.

The need for highly structured individualised programmes was borne out by the pilot project, the feedback workshops and the concurrent development of a structured therapy programme within the service. This information manifested itself in three distinct areas:

- structured professional judgement and risk

- individual care plan

- pillars of care.

\section{Cycle 3 - structured professional judgement and risk}

Risk assessment and management are now considered crucial for determining the needs of the patient, and in order that optimum levels of care can be provided, reducing the potential for negative outcomes and allowing positive risk taking (Kennedy, 2000). The culture of using structured professional judgement and semi-structured risk assessment tools was already established within the service. This previous work and training provided a framework for the steering group to agree the various assessments and time scales. A risk assessment book was compiled in Cycle 1, incorporating each of the assessments, but was withdrawn due to lack of completion.

Further consultation identified fragmented working practices in conducting risk assessments and equally an over-abundance of paperwork. Assessments were being completed, but not filed in this system, and agreement was reached on having individual risk assessments available for viewing by relevant personnel on the service's computer system. At this stage the ICP process began a parallel approach of electronic and paper systems, which will be referred to later.

This process of elimination did, however, lead clinicians to challenge traditional practices in which risk assessment was carried out without much user involvement. Use of the HCR-20 (Webster et al, 1997) and the structure that it provides is not only considered to help clinicians in relation to understanding risk, but has also proved therapeutically beneficial when discussed and shared with the patient (Webster et al, 2004). From the service user perspective, it removes some of the mystery from the risk assessment process and provides greater awareness of risk issues before their tribunals and other decision-making forums.

Documenting the evidence for each item of the risk assessment in the ICP provides a focus for both the patient and the treating team, enabling discussion of particular risks. This ensures that those risks are addressed in the care plan. Identifying protective factors for various risk items with the service user ensures that service user strengths are not overlooked and are incorporated into the pathway by documenting in the service user's care plan. This provides the opportunity to identify discrepancies and gaps in information and, even where there is disagreement, it allows the possibility of discussing the same. This discussion can then inform the sequencing of programming for each area of care. 


\section{Pillars and pathways: foundations of recovery in Irish forensic mental health care}

The use of structured professional judgement tools has provided a clear focus for both the service user and the clinician in relation to risk management plans. The dynamic items in the risk assessments are ways in which progress can be measured and shared with the service user. Areas where change is difficult can be further examined to identify future interventions. Service users have generally viewed risk assessment in a negative way, and usually associated it with their continuing detention. However, linking the risk items from these assessments directly to the individual's care plan is now thought to provide transparency for the service user and clinicians alike. As such, it has become an integral part of the ICP.

\section{Cycle 4 - individual care plan}

The literature review identified clearly that any care pathway should form all or part of the clinical record and document clearly the care given (De Bleser et al, 2006; Green, 2008). The importance of agreeing a set of minimum standards to ensure appropriate and timely interventions was identified (Kent \& Chalmers, 2006). It was also acknowledged that variations from best practice and gaps in care or unmet need should be identified and recorded in order to develop a culture of continuous quality improvement. More important, the care pathway should be recovery-focused for the individual service user, based on their assessed need and risk.

Through a series of multi-disciplinary workshops it was considered that this information could be best gathered by a single individual care plan. A care plan book was developed in Cycle 1 and piloted, but met with a poor response. Clinical standards had been formulated following feedback from service users' and carers' satisfaction surveys, and direct consultation with the residents' forum and the carers' group. Again, the quantity of paperwork became an issue, as did the reluctance of professionals to use a structured format in a ward round setting. The concept of primary nursing was still new during the pilot phase of this project, and there were significant MDT staff shortages in one of the teams in the acute cluster. Likewise the notion of patients being actively involved in their care planning remained an untested value for some clinicians. There were further concerns about the loss of the nursing process in an individual care plan.

An audit of the ICP took place in May 2009. Although the care plan was poorly completed, there was a highly structured and well-recorded case conference process, and a very early process for capturing treatment outcomes. The case conference had been set clearly in a time frame by the teams, and had a clear structure. A continued weakness is the omission of the patient's views on why they have been admitted to the service. This requires further exploration, as indeed does the need for a dynamic and live care plan that reflects the daily and weekly lives of our patients between case conferences. A second draft has been developed for further discussion with key stakeholders.

\section{Cycle 5 - pillars of care}

Throughout this process and in discussion with all stakeholders it became increasingly obvious that the ICP could only be truly effective if a range of evidence-based treatments were available, and in a timescale that facilitated transition of patients through the service. On further consultation and with due consideration of our needs analysis, the notion of looking at developing key standards for key areas of care was discussed. It was considered essential that the care pathway provide transparency for staff, service users and carers, by clearly linking needs and risk assessment to particular evidence-based interventions.

One pragmatic manner of managing what had become a leviathan of therapies, treatments, and vocational and occupational activities was to develop a pathway across agreed 'pillars of care'. These pillars are outlined in Table 2, below. After further consultation and a review of the literature on what works for each pillar, individual items were grouped together that addressed similar needs and/ or risks. This process initially focused on three main strands of:

- illness, insight awareness and recovery

- substance misuse

- offending behaviour.

However, on further reflection, it was considered that the process should be extended to five pillars, to further address physical health care and psychosocial functioning. The title of 'offending behaviour' was also renamed 'harmful behaviour' to reflect those patients who have not come into contact with the criminal justice system.

A key cornerstone of the success of these pillars was agreeing a set of key clinical standards that could then be structured within an individual care plan. Draft 2 of the ICP has therefore been structured into these five pillars of care. Each pillar is intended to classify a series of common needs and/or risks and the requisite assessments 
Table 2: Labelling pillars of care

\begin{tabular}{lllll}
\hline Pillar 1 & Pillar 2 & Pillar 3 & Pillar 4 & Pillar 5 \\
\hline $\begin{array}{l}\text { Physical } \\
\text { treatment }\end{array}$ & $\begin{array}{l}\text { Illness, insight, } \\
\text { wellness and } \\
\text { recovery }\end{array}$ & $\begin{array}{l}\text { Drugs and } \\
\text { alcohol }\end{array}$ & $\begin{array}{l}\text { Harmful } \\
\text { behaviour }\end{array}$ & $\begin{array}{l}\text { Psychosocial, } \\
\text { occupational } \\
\text { and rehabilitation }\end{array}$
\end{tabular}

and interventions that should be considered to address these needs/risks. It is not anticipated that all these pillars will be addressed concurrently or at one single point in time. These pillars map a pathway of care that should be addressed before the patient is discharged. These pillars become central to the tenet of recovery within our service, where strengths are more commonly identified than deficits and where a more holistic delivery of care is available to the patient.

\section{Conclusion}

The development of an ICP within the National Forensic Mental Health Service has afforded us the opportunity to examine many aspects of the care delivered in the service. It has provided a shared understanding between clinicians, service users and carers of the key standards that are necessary to implement a quality care pathway. It has certainly not been a stagnant process, and the initial documentation often bears no resemblance to the current process. In turn, we expect that it will continue to change, as the path travelled is as important as the outcome, and the ICP becomes a dynamic part of the organisation.

The development of the pillars of care developed from this review, and has identified the advantages of using action research principles in seeking to introduce significant change in service delivery. Likewise, the involvement of so many people within the action research process itself has helped unlock a number of traditional practices, highlighting that knowledge is derived from practice, and practice is informed by knowledge, in an ongoing cyclical process.

For service users, explicitly linking various items in the risk and need assessment tools to the individual's care provided a clear link between risk and care planning. This enabled the service user to identify their needs, strengths and risks, and provided clarity and hope in working towards their recovery. Inevitably, not all service users, carers or indeed clinicians were directly consulted, and there is room for improvement as the process is developed further.
In any event, the NFMHS has joined the growing ranks of organisations attempting to use an ICP approach to provide a framework in which to improve clinical outcomes, cost-effectiveness, patient involvement and clinical risk management. The next step is to continue this pathway across the remainder of the service.

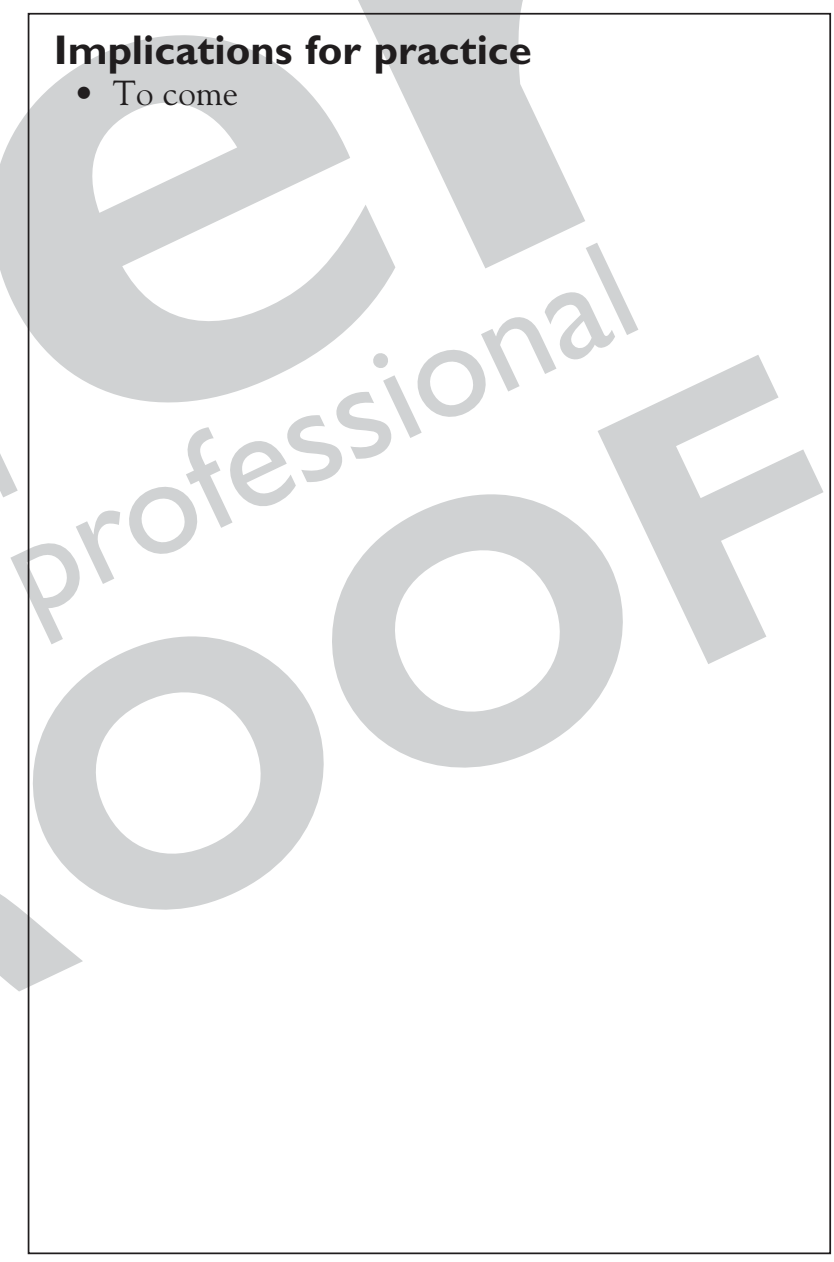

\section{Address for correspondence}

Pauline Hill

National Forensic Mental Health Service

Dublin

Ireland

pauline.gill@hse.ie

david.timmons@hse.ie 


\section{Pillars and pathways: foundations of recovery in Irish forensic mental health care}

\section{References}

Adshead G \& Sarkar SP (2005) Justice and welfare: two ethical paradigms in forensic psychiatry. Australian and New Zealand Journal of Psychiatry 39 (11-12) 1011.

Atwal A \& Caldwell K (2002) Do multidisciplinary integrated care pathways improve inter-professional collaboration? Scandinavian Journal of caring Science 16 $360-67$.

Badger D, Vaughan P, Woodward M \& Williams P (1999) Planning to meet the needs of offenders with mental disorders in the United Kingdom. American Psychiatric Association 50 (12) 1624-7.

Bouch J \& Marshall J (2003) S-RAMM. Suicide Risk Assessment Management Manual. Glamorgan: The Cognitive Centre Foundation.

Criminal Law (Insanity) Act (2006). Dublin Stationery Office.

De Bleser L, Depreitere R, De Waele K et al (2006) Defining pathways. Journal of Nursing Management 14 553-63.

Department of Health and Children (2006) A Vision for Change: Report of the Expert Group on Mental Health Policy. Dublin: Stationery Office.

Gralton E, Udu V \& Ranasinghe S (2006) A solutionfocused model and inpatient secure settings. British Journal of Forensic Practice 8 (1) 24-30.

Green B (2008) Implementing and using care pathways in mental health: developing an approach for practice. Journal of Management and Marketing in Healthcare 1 (3) $231-42$.

Hayward-Rowse L \& Whittle T (2006) A pilot project to design, implement and evaluate an electronic integrated care pathway. Journal of Nursing Management 14 564-71.

Kalliola S (2009) Learning along with participatory action research - a Finnish perspective. International Journal of Action Research 5 (3) 289-321.

Kennedy H (2000) Therapeutic uses of security: mapping forensic mental health services by stratifying risk. Advances in Psychiatric Treatment 8 433-43.

Kent P \& Chalmers Y (2006) A decade on: has the use of integrated care pathways made a difference in Lanarkshire? Journal of Nursing Management 14 508-20.
Lamont S, Walker P \& Brunero S (2009) Teaching an old dog new tricks. Practice Development in Healthcare 8 (2) $65-76$.

Madden T (2007) Treating Violence - A guide to risk management in mental health. Oxford: Oxford University Press.

Mental Health Act (2001) Dublin: Stationery Office.

Mental Health Act (2006) Mental Health Act 2001 (Approved Centres) Regulations 2006.

Mental Health Commission (2009) Code of Practice on Admission, Transfer and Discharge to and from an Approved Centre. Dublin: MHC.

Ogloff J \& Daffern M (2006) the dynamic appraisal of situational aggression: an instrument to assess risk for imminent aggression in psychiatric inpatients. Behavioural Sciences and the Law 24 799-813.

O'Neill C, Heffernan P, Goggins R et al (2003) Long stay forensic psychiatric inpatients in the Republic of Ireland: aggregated needs assessment. Irish Journal of Psychiatric Medicine 20 (4) 119-25.

Palmer V (2009) Getting with the act of action research. Forum: Qualitative Social Research 10 (3) 26.

Riley K (1998) Paving the way. Health Service Journal 108 30-31.

Slade M, Powell R, Rosen A \& Strathdee G (2000) Threshold Assessment Grid (TAG): the development of a valid and brief scale to assess the severity of mental illness. Social Psychiatry and Epidemiology 35 78-85.

Thomas S, Harty M, Parrot J et al (2003) CANFOR: Camberwell Assessment of Need - Forensic Version. London: Gaskell.

Webster C, Douglas K, Eaves D \& Hart S (1997) HCR20 Assessing risk for violence. Mental Health, Law and Policy Institute. Canada: Simon Fraser University.

Webster C, Martin M, Brink J, Nicholls T \& Middleton C (2004) Short-Term Assessment of Risk and Treatability (START): An evaluation and planning guide. British Columbia: Forensic Psychiatric Services Commission.

Zander K (2002) Integrated care pathways: eleven international trends. Journal of Integrated Care Pathways 6 $101-7$. 


\title{
Long-term care needs in male medium security
}

John Jacques

CONSULTANT FORENSIC PSYCHIATRIST

Sarah-Jane Spencer SPR IN FORENSIC PSYCHIATRY

THE ORCHARD, WEST LONDON MENTAL HEALTH NHS TRUST, UK

Paul Gilluley CONSULTANT FORENSIC PSYCHIATRIST, THREE BRIDGES MEDIUM SECURE UNIT, WEST LONDON MENTAL HEALTH NHS TRUST, UK

\begin{abstract}
Medium secure units were designed to treat patients for up to three years, but some patients spend longer in acute medium secure settings which in general do not have a 'longer term focus'. The aim of this investigation was to assess and describe the needs of these patients. A survey questionnaire was designed and sent to responsible clinicians who had patients admitted at least five years previously to the Three Bridges Medium Secure Unit (males) in West London. Carer ratings using the Camberwell Assessment of Need (forensic version: CAN-FOR) was completed by the primary nurse for each patient, complementing the survey questionnaire. Of 122 medium secure male patients 25 (21\%) had been admitted at least five years before. High levels of co-morbidity were found, as well as treatment resistance. The CAN-FOR revealed two groups, one with chronic challenging behaviour, treatment-resistant mental illness and need for a high level of support, and another more able group not needing as much support but with a dependency on the hospital. It is considered here whether certain groups would benefit from a different approach or setting.
\end{abstract}

\section{KEY WORDS}

medium secure; long term; rehabilitation; service development

\section{Introduction}

Medium secure services were developed following the Butler Report (Home Office, DH \& DSSS, 1975), which recommended provision of 2000 medium secure beds for mentally disordered offenders in England and Wales. The target length of stay in medium security was originally 18 months to two years, and the units were designed with this 